\title{
Associations of Testosterone and Sex Hormone Binding Globulin with Adipose Tissue Hormones in Midlife Women
}

\author{
Rachel P. Wildman ${ }^{1}$, Dan Wang ${ }^{1}$, Ivonne Fernandez ${ }^{2}$, Peter Mancuso ${ }^{3}$, Nanette Santoro ${ }^{4}$, \\ Philipp E. Scherer ${ }^{5}$ and MaryFran R. Sowers ${ }^{6^{*}}$
}

Objective: Regulators of adipose tissue hormones remain incompletely understood, but may include sex hormones. As adipose tissue hormones have been shown to contribute to numerous metabolic and cardiovascular disorders, understanding their regulation in midlife women is of clinical importance. Therefore, we assessed the associations between testosterone (T) and sex hormone binding globulin (SHBG) with leptin, high molecular weight (HMW) adiponectin, and the soluble form of the leptin receptor (sOB-R) in healthy midlife women.

Design and Methods: Cross-sectional analyses were performed using data from 1,881 midlife women (average age $52.6( \pm 2.7)$ years) attending the sixth Annual follow-up visit of the multiethnic Study of Women's Health Across the Nation.

Results: T was weakly negatively associated with both HMW adiponectin and sOB-R $(r=-0.12$ and $r=$ -0.10 , respectively; $P<0.001$ for both), and positively associated with leptin $(r=0.17 ; P<0.001)$. SHBG was more strongly and positively associated with both HMW adiponectin and sOB-R $(r=0.29$ and $r=0.24$, respectively; $P<0.001$ for both), and more strongly and negatively associated with leptin $(r$ $=-0.27 ; P<0.001$ ). Adjustment for fat mass, insulin resistance, or waist circumference only partially diminished associations with HMW adiponectin and sOB-R, but attenuated associations with leptin. In conclusion, in these midlife women, lower SHBG values, and to a lesser extent, higher $\mathrm{T}$ levels, were associated with lower, or less favorable, levels of adiponectin and SOB-R, independent of fat mass.

Conclusions: These data suggest that variation in these adipose hormones resulting from lower SHBG levels, and possibly, though less likely, greater androgenicity, may contribute to susceptibility for metabolic and cardiovascular outcomes during midlife in women.

Obesity (2013) 21, 629-636. doi:10.1002/oby.2012.109

\section{Introduction}

Adipose tissue is well-recognized as an endocrine organ, which secretes a number of hormones. Two such hormones, adiponectin and leptin, are secreted solely by adipose tissue, and have been implicated in metabolic and cardiovascular complications. Adiponectin is lower among obese individuals, and possesses anti-inflammatory, antidiabetic, and antiatherogenic properties. Leptin is higher among obese individuals, thought to be due to leptin resistance, and is associated with a pro-inflammatory, atherogenic milieu. Regulators of adipose tissue-derived hormones remain poorly understood. While fat mass cer- tainly regulates adipose tissue hormone levels, it is not the only regulator, evidenced by the fact that adipose hormone levels vary considerably in people of similar fat mass. Men have less favorable adipose hormone profiles than women, even after accounting for differences in fat mass $(1,2,3)$, raising the question of whether androgen levels may regulate adipose hormone levels. Testosterone $(\mathrm{T})$ administration in men has been shown to decrease both adiponectin and leptin levels $(4,5)$, and adipose hormone levels in women with polycystic ovary syndrome (PCOS), a syndrome whose hallmark is hyperandrogenemia, have been well-characterized, with similar leptin levels but lower adiponectin levels compared with BMI-matched controls $(6,7)$.

\footnotetext{
${ }^{1}$ Department of Epidemiology and Population Health, Albert Einstein College of Medicine, Bronx, New York, USA. *Deceased. Correspondence: Rachel P. Wildman (Rachel.wildman@einstein.yu.edu) ${ }^{2}$ Department of Internal Medicine, Englewood Hospital, Affiliate of Mount Sinai School of Medicine, Englewood, New Jersy, USA ${ }^{3}$ Department of Environmental Health Sciences, School of Public Health, University of Michigan, Ann Arbor, Michigan, USA ${ }^{4}$ Department of Medicine, University of Colorado School of Medicine, Aurora, Colorado, USA ${ }^{5}$ Touchstone Diabetes Center,Departments of Internal Medicine and Cell Biology, The University of Texas Southwestern Medical Center, Dallas, Texas, USA ${ }^{6}$ Department of Epidemiology, School of Public Health, University of Michigan, Ann Arbor, Michigan, USA
}

Disclosure: The authors declared no conflict of interest.

See the online ICMJE Conflict of Interest Forms for this article.

Received: 9 September 2011 Accepted: 29 March 2012 First published online by Nature Publishing Group on behalf of The Obesity Society 24 May 2012. doi:10.1002/oby.2012.109 
Prior studies of the associations between sex hormones and adipose tissue hormones have overwhelmingly focused on PCOS women, who tend to be obese and metabolically dysregulated, leaving the understanding of regulators of adipose hormones in normal weight women relatively unexplored. As some evidence suggests that associations between androgens and adipose hormones may differ in normal weight vs. obese individuals $(8,9,10)$, examination of the potential modifying role of fat mass on androgen-adipose hormone associations is needed. In addition to being few in number, previous studies in healthy women have adjusted for BMI, a poor proxy for adiposity levels, and have suffered from relatively small sample sizes and failure to account for potential confounders such as accompanying insulin resistance levels. In addition, associations between androgens and soluble leptin receptor (sOB-R), the primary leptin-carrier protein in the circulation and thus, a potential regulator of leptin's effects, have not been examined in healthy women. Therefore, the purpose of the current study was twofold: (i) to examine the associations between T and SHBG with leptin, high molecular weight (HMW) adiponectin, and sOB-R and(ii) to examine whether these associations differed in nonobese vs. obese women. These associations were examined in midlife women participating in the Study of Women's Health Across the Nation (SWAN).

\section{Methods and Procedures}

\section{Participants}

SWAN is a multi-center, longitudinal study to characterize the biological and psychosocial changes with the menopause in a community-based sample. SWAN sites are Boston, Chicago, Detroit, Los Angeles, Newark, Pittsburgh, and Oakland CA. From 1996 to 1997, 3,302 women aged 42-52 years were enrolled. Each site recruited white women plus one racial/ethnic group including white, African American (Pittsburgh, Michigan, and Boston), Chinese (Oakland), Japanese (UCLA) and Hispanic (Newark-Central American, South American, and Caribbean origin) women. Complete information on screening and data collection has been published previously (11). Briefly, at SWAN baseline, women had an intact uterus and were still menstruating, had at least one ovary and were not pregnant or breastfeeding. Exclusion criteria included oral contraceptive or sex steroid hormone therapy use in the prior 3 months. Institutional review board approval and informed consent was obtained.

With the exception of race-ethnicity and highest grade level achieved, which were assessed at the SWAN baseline examination, the current report uses sex hormone, adipose tissue hormone, and covariate data collected at the sixth annual follow-up visit, the only visit at which adipose hormones were available on the full SWAN cohort. Among the 2,441 women who attended the sixth annual visit, blood samples were available and adipose hormone data were able to be assayed on 2,028 women, with 1,950 having data on all three adipose hormones of interest. After excluding 69 women missing key covariates of interest, data from 1,881 women were available for the current analyses.

\section{Body size and composition measures}

Height and weight were measured in light clothing without shoes and using calibrated scales. BMI was calculated as weight in kilograms divided by height in $\mathrm{m}^{2}$. Waist circumference was measured in nonrestrictive undergarments, or in cases where respondents refused, measures were taken over light clothing. Waist circumference was measured at the level of the natural waist, defined as the narrowest part of the torso as seen from the anterior aspect. In cases where a waist narrowing was difficult to identify, the measure was taken at the smallest horizontal circumference in the area between the ribs and the iliac crest. Fat mass was estimated from bioelectrical impedance analysis (BIA) (BIA-103 analyzer; RJL Systems, Mt. Clemens, MI). BIA is based on measurement of the conductivity of an electrical pulse between electrodes attached at the feet and the knuckles of the hand. Electrical conductivity is greater in fat-free than in fat mass, and thereby resistance and reactance can be used to estimate fat and lean mass $(12,13)$. Sex-specific validation equations of Chumlea et al. were used (14).

\section{Questionnaire data}

Menopause status was assessed based on menstrual bleeding and use of hormone therapy as follows:

- Pre- or early-perimenopause: monthly bleeding with or without a perceived change in cycle interval, but at least one period within the past 3 months.

- Late perimenopause: $\geq$ three consecutive months of amenorrhea.

- Post menopause: $\geq 12$ consecutive months of amenorrhea.

- Surgical menopause: menopause induced by hysterectomy with or without oophorectomy

- Unknown: use of menopausal hormone therapy (MHT) among pre- or early-perimenopasual women

Smoking was assessed by questionnaire and coded as never, past, or current. Physical activity was based on the Kaiser Permanente Activity Score (15).

\section{Assays}

Fasting blood draws were targeted to the follicular phase of the menstrual cycle (days 2-5) and maintained at $4{ }^{\circ} \mathrm{C}$ until separated and frozen at $-80{ }^{\circ} \mathrm{C}$. T, sex hormone binding globulin (SHBG), and estradiol were assayed at the University of Michigan Endocrine Laboratory using an ACS-180 automated chemiluminescence analyzer (Bayer Diagnostics, Norwood, MA), with modifications to enhance sensitivity in the low ranges. To avoid disproportionate exclusion of women with low hormone values, imputation of hormone values below the lower limit of detection ( $n=8$ for SHBG and $n=3$ for T) was performed (16), such that a random value between zero and the lower limit of detection was used for these women.

Leptin, sOB-R, and HMW adiponectin were determined in the laboratory of Dr. Peter Mancuso in duplicate using commercially available colorimetric enzyme immunoassay kits according to the manufacturer's instructions (leptin and HMW adiponectin; Millipore, St. Charles, MO and sOB-R; R\&D systems, Minneapolis, MN). The mean coefficient of variation percent for duplicate samples for each subject and lower limit of detection, respectively, were HMW adiponectin: $8.1 \%, 0.5 \mathrm{ng} / \mathrm{ml}$; leptin: $4 \%, 0.5 \mathrm{ng} / \mathrm{ml}$; and sOB-R: $3.7 \%, 0.31 \mathrm{ng} / \mathrm{ml}$.

Serum insulin level was measured using a solid-phase radioimmunoassay (DPC Coat-A-Count Insulin RIA; Diagnostic Products, Los Angeles, CA). Glucose levels were measured using a hexokinasecoupled reaction (Boehringer Mannheim Diagnostics, Indianapolis, IN). The homeostasis model assessment insulin resistance index (HOMA-IR) was calculated from fasting insulin and glucose as (fasting insulin $(\mathrm{mU} / \mathrm{l}) \times$ fasting glucose $(\mathrm{mmol} / \mathrm{l})) / 22.5$.

\section{Statistical methods}

Characteristics of the study population were summarized as frequency (\%), mean (s.d.) for normally distributed variables, or as 
median (interquartile range) for skewed variables in the overall sample as well as in nonobese (BMI $<30 \mathrm{~kg} / \mathrm{m}^{2}$ ) and obese (BMI $\geq 30$ $\mathrm{kg} / \mathrm{m}^{2}$ ) women, separately. $\chi^{2}$ tests for categorical variables, $t$-tests for normally distributed continuous variables, and Wilcoxon tests for skewed continuous variables were used to evaluate whether differences in characteristics between nonobese and obese women were statistically significant.

Median (interquartile range) adipose hormone values were calculated by quartiles of testosterone and SHBG. $P$ values for linear trend across quartiles were calculated using the median sex hormone value in each quartile as a continuous variable in the context of linear regression modeling, whereby log-transformed adipose hormones were dependent variables.

Partial Spearman correlation coefficients were then calculated for associations between testosterone, and SHBG with adipose tissue hormones, initially adjusting for age, race-ethnicity, site, cycle day of blood draw, menopausal status, physical activity, smoking status, and education level. Additional adjustments were made for fat mass, HOMA-IR, or waist circumference. To insure that SHBG was not acting as a proxy for estradiol, further adjustment of the correlation coefficient was also made for estradiol. Correlation coefficients were calculated for each sex hormone and each adipose hormone separately. Following this, for each adipose hormone, partial correlation coefficients were adjusted for values of the other sex hormone, and separately for values of the other adipose hormones.

To examine whether associations between testosterone and SHBG with adipose tissue hormones differed by body size, stratified analyses were performed whereby partial correlation coefficients between testosterone and SHBG with adipose tissue hormones were calculated in obese and nonobese women, separately. To formally evaluate potential effect modification, multiplicative interaction terms were tested for logged values of testosterone and SHBG with a categorical BMI variable $\left(<30 \mathrm{~kg} / \mathrm{m}^{2}\right.$ (nonobese) vs. $\geq 30 \mathrm{~kg} / \mathrm{m}^{2}$ (obese) $)(\log ($ SHBG $) \times$ BMI category and $\log (\mathrm{T}) \times$ BMI category $)$ in the context of log-adipose hormone linear regression models. Similar interaction terms were tested with race-ethnicity and menopausal status to evaluate effect modification by each of these.

Sensitivity analyses examined the consistency of the findings when women reporting hysterectomy or MHT use were excluded, and when BMI was adjusted for instead of fat mass. Additional sensitivity analyses repeated calculation of correlation coefficients excluding the 82 women with a high probability of PCOS, per their designation as having hyperandrogenemic oligomenorrhea, defined as having total testosterone values in the highest tertile of the cohort distribution, and a reported history of oligomenorrhea, defined as $\geq 3$ months without a menstrual period between the ages of 25 and 35 years in the context of a negative report of pregnancy, breastfeeding, or birth control use during those months, and exclusion of other causes such as abnormal thyroid stimulating hormone using National Institutes of Health criteria.

\section{Results}

By the sixth annual follow-up visit at which these cross-sectional analyses took place, SWAN women were an average of 52.6 years old, 51\% were white, 28\% African American, 9\% Chinese, 11\% Japanese, and $<1 \%$ Hispanic (Table 1). Over one-third of women were still premenopausal or early-perimenopausal and were not using MHT, while close to $50 \%$ had undergone surgical or natural menopause, and $8 \%$ reported MHT use since the last study visit. When stratified by body size, obese women (BMI $\geq 30 \mathrm{~kg} / \mathrm{m}^{2}$ ) were more often African American, had lower education levels, were less often still pre- or early-perimenopausal, and had higher fat mass, waist circumference, HOMA, testosterone, and leptin values; and lower SHBG, sOB-R, and HMW adiponectin values compared with nonobese women (BMI $<30 \mathrm{~kg} / \mathrm{m}^{2}$; Table 1).

HMW adiponectin and sOB-R were both negatively correlated with BMI, fat mass, and waist circumference (Supplementary Table S1 online; Spearman correlation coefficients ranging from -0.33 to -0.43 ; all $P<0.001$ ), while leptin levels were positively correlated with indices of body size and adiposity (correlation coefficients ranging from 0.82 to 0.86 ). In addition, each of the adipose tissue hormones was significantly associated with the others, with HMW adiponectin significantly positively associated with sOB-R (correlation coefficient $0.45, P<0.001$ ), and significantly negatively correlated with leptin (correlation coefficient $-0.34 ; P<0.001$ ), and leptin and sOB-R similarly negatively correlated (correlation coefficient $-0.42 ; P<0.001)$.

With higher testosterone quartiles, HMW adiponectin and sOB-R levels were lower, whereas leptin levels were higher (Table 2). Opposite results were found with higher SHBG quartiles. After multivariate adjustment which included not only age, race-ethnicity, menopausal status, physical activity, smoking status, education level, cycle day of blood draw, and study site, but also fat mass, and subsequent models including waist circumference or HOMAIR, HMW adiponectin levels were lower and leptin levels higher with higher testosterone levels, though the magnitude of associations were considerably reduced with adjustment for fat mass or HOMA-IR (Table 3). The magnitude of correlation coefficients was similar after further adjustment of testosterone results for SHBG.

After initial adjustment for age, race-ethnicity, menopausal status, physical activity, smoking status, education level, cycle day of blood draw, and study site, SHBG was positively associated with HMW adiponectin and sOB-R, and negatively associated with leptin. As above, the association with leptin was substantially attenuated with adjustment for fat mass. However, the positive associations between SHBG and both HMW adiponectin and sOB-R were quite robust to additional adjustment not only for fat mass, but also for HOMA-IR, waist circumference, and testosterone. Results were also additionally adjusted for estradiol to insure that SHBG was not acting as a proxy for estradiol, and results were, again, similar (partial correlation coefficients of 0.24 and 0.17 for HMW adiponectin and sOB-R, respectively, after additional adjustment for estradiol; both $P<$ $0.001)$.

When all three adipose hormones were also adjusted for each other (Table 3, partial 6), HMW adiponectin results were only slightly attenuated. The positive association between testosterone and leptin was unchanged, while the negative association between SHBG and leptin was only slightly attenuated, but lost statistical significance. The opposite finding was true for $\mathrm{SOB}-\mathrm{R}$; the negative association with testosterone was only slightly attenuated but lost statistical 
TABLE 1 Characteristics of the study population at the sixth annual SWAN visit

\begin{tabular}{|c|c|c|c|c|}
\hline Variable & Overall $(n=1,881)$ & $\mathrm{BMI}<30 \mathrm{~kg} / \mathrm{m}^{2}(n=1,179)$ & $\mathrm{BMI} \geq 30 \mathrm{~kg} / \mathrm{m}^{2}(n=655)$ & $P$ \\
\hline Age, years & $52.6(2.7)$ & $52.5(2.6)$ & $52.6(2.7)$ & 0.409 \\
\hline Race-ethnicity, $n(\%)$ & & & & $<0.001$ \\
\hline White & $951(51)$ & $609(52)$ & $321(49)$ & \\
\hline African American & $535(28)$ & $205(17)$ & $304(46)$ & \\
\hline Chinese & $175(9)$ & $162(14)$ & $13(2)$ & \\
\hline Japanese & $215(11)$ & $199(17)$ & $16(2)$ & \\
\hline Hispanic & $5(<1)$ & $4(0.3)$ & $1(0.2)$ & \\
\hline Current smokers, $n(\%)$ & $240(13)$ & $140(12)$ & $89(14)$ & 0.288 \\
\hline Education level, $n(\%)^{a}$ & & & & $<0.001$ \\
\hline Less than high school & $344(18)$ & $193(16)$ & $141(22)$ & \\
\hline Post high school & $620(33)$ & $347(30)$ & $253(39)$ & \\
\hline College and higher & $906(49)$ & $634(54)$ & $256(39)$ & \\
\hline Menopause status, $n(\%)$ & & & & 0.016 \\
\hline Pre/early-perimenopausal & $613(33)$ & $401(34)$ & $199(30)$ & \\
\hline Late perimenopausal & $193(10)$ & $107(9)$ & $83(13)$ & \\
\hline Postmenopausal/surgical menopause & $925(49)$ & $568(48)$ & $331(51)$ & \\
\hline MHT user & $150(8)$ & $103(9)$ & $42(6)$ & \\
\hline Fat mass, $\mathrm{kg}$ & $30.1(13.7)$ & $22.0(6.5)$ & $44.7(10.5)$ & $<0.001$ \\
\hline $\mathrm{BMI}, \mathrm{kg} / \mathrm{m}^{2}$ & $28.8(7.4)$ & $24.3(3.1)$ & $36.8(5.9)$ & $<0.001$ \\
\hline Waist circumference, $\mathrm{cm}$ & $88.9(16.8)$ & $79.2(8.8)$ & $105.9(12.7)$ & $<0.001$ \\
\hline HOMA insulin resistance ${ }^{\mathrm{b}}$ & $2.2(1.9)$ & $1.8(1.1)$ & $3.4(3.0)$ & $<0.001$ \\
\hline Testosterone, ng/dl $(0.0347 \mathrm{nmol} /)^{\mathrm{b}}$ & $36.4(23.2)$ & $34.5(20.9)$ & $39.7(24.6)$ & $<0.001$ \\
\hline $\mathrm{SHBG}, \mathrm{nmol} / \mathrm{l}^{\mathrm{b}}$ & $41.3(32.8)$ & $47.2(36.0)$ & $33.0(23.9)$ & $<0.001$ \\
\hline Leptin, ng/m/ $\left.\right|^{b}$ & $22.8(28.3)$ & $14.7(14.3)$ & $44.4(27.3)$ & $<0.001$ \\
\hline $\mathrm{sOB}-\mathrm{R}, \mathrm{ng} / \mathrm{m}^{\mathrm{b}}$ & $29.3(12.7)$ & $31.9(12.8)$ & $25.2(9.8)$ & $<0.001$ \\
\hline HMW adiponectin, $\mu \mathrm{g} / \mathrm{ml}^{\mathrm{b}}$ & $5.4(6.3)$ & $6.8(7.0)$ & $3.8(4.3)$ & $<0.001$ \\
\hline
\end{tabular}

significance, while the positive association with SHBG was slightly attenuated, but remained statistically significant.

We also examined effect modification of these associations by body size, race-ethnicity, and menopausal status in models adjusted for the factors listed above as well as fat mass. Significant interaction terms were found for body size concerning the associations between SHBG with both leptin $(P$-interaction $<0.001)$ and sOB$\mathrm{R}(P$-interaction $=0.025)$. For leptin, a negative association was found with SHBG in nonobese participants (Table 4; $r=-0.11$ ), but a weak positive association in obese participants (Table $4 ; r=$ 0.08). For sOB-R, a positive association was found with SHBG in nonobese participants (Table 4; $r=0.19$ ), but a null association in obese participants (Table $4 ; r=0.05$ ). Body size interaction terms were not statistically significant for associations between $\mathrm{T}$ and any of the adipose tissue hormones, or between SHBG and HMW adiponectin.

Significant interaction terms were also found for race-ethnic effects on the associations between SHBG and leptin $(P=0.006)$, and testosterone and sOB-R $(P=0.009)$. When stratified by race-ethnicity, partial correlation coefficients between SHBG and leptin were similar in magnitude among African American and white women $(r=$ 0.02 and -0.06 , respectively), but were stronger in Chinese and Japanese women $(r=-0.10$ and -0.18 , respectively). For the association between testosterone and sOB-R, it appeared to be null for all, but the Japanese, in whom it was negative $(r=-0.13)$. Significant interactions were not detected for menopausal status, indicating that associations between sex hormones and adipose tissue hormones were similar within each of the menopause status categories. 
Results were similar when women reporting hysterectomy or MHT use were excluded from analyses, when BMI was adjusted for instead of fat mass, and when the 82 suspected PCOS cases were excluded.

\section{Discussion}

Testosterone receptors have been found in various tissues, including adipose tissue (17), raising the possibility that testosterone may serve as a regulator of adipose tissue hormones. SHBG is a liver-derived binding and transport protein for both testosterone and estradiol, though it has greater affinity for testosterone (18). However, in addition to binding sex steroids and, therefore, determining the amount of free steroid, SHBG has also been shown to bind to membrane receptor sites, itself, raising the possibility that SHBG may have direct effects on cell signaling independent of its influence on the biologic activity of estradiol and testosterone (19). Therefore, like testosterone, SHBG is also a potential regulator of adipose tissue hormone levels. However, few investigations have examined the relationships between testosterone or
SHBG and adipose tissue hormones in healthy women. Among these midlife women, we found that higher testosterone levels and lower SHBG levels were associated with lower HMW adiponectin and sOB-R levels, and higher leptin levels, independent of levels of fat mass, and in most cases, independent of HOMA-IR and waist circumference as well. Therefore, our data support the possibility that SHBG and testosterone may regulate adipose tissue hormones.

The strongest associations documented in these midlife women were between SHBG and both HMW adiponectin and sOB-R, whereby even after adjustment for fat mass, HOMA-IR, waist circumference, testosterone, or estradiol partial correlation coefficients were $\sim 0.20$ for HMW adiponectin and 0.15 for sOB-R. In agreement with our findings, several studies have documented a significant positive association between SHBG and adiponectin (20-23). To our knowledge, prior published reports have not examined the association between SHBG and sOB-R. SOB-R is the primary carrier protein for leptin in the blood stream, and is a cleaved derivative of membrane-anchored leptin receptors. The scope of the physiologic role of sOB-R is still largely unknown. Higher sOB-R levels may

TABLE 2 Median (25th percentile, 75th percentile) adipose hormone values by quartiles of testosterone and SHBG

\begin{tabular}{|c|c|c|c|c|c|}
\hline Leptin, ng/ml & $17.7(9.1,31.3)$ & $22.2(11.9,37.4)$ & $22.0(12.3,40.4)$ & $31.1(16.5,47.6)$ & $<0.001$ \\
\hline $\mathrm{sOB}-\mathrm{R}, \mathrm{ng} / \mathrm{ml}$ & $30.9(24.9,37.4)$ & $29.1(24.1,37.3)$ & $29.2(23.6,35.9)$ & $27.4(21.8,34.2)$ & $<0.001$ \\
\hline HMW adiponectin, $\mu \mathrm{g} / \mathrm{ml}$ & $3.8(2.1,7.2)$ & $5.0(2.7,8.3)$ & $5.9(3.5,9.8)$ & $7.4(4.2,11.6)$ & $<0.001$ \\
\hline Leptin, ng/ml & $30.2(17.5,45.1)$ & $25.8(14.7,45.6)$ & $21.0(11.8,36.0)$ & $14.8(7.5,28.5)$ & $<0.001$ \\
\hline $\mathrm{sOB}-\mathrm{R}, \mathrm{ng} / \mathrm{ml}$ & $27.3(22.1,33.1)$ & $27.3(22.4,33.7)$ & $30.1(24.2,36.7)$ & $33.1(27.1,40.5)$ & $<0.001$ \\
\hline
\end{tabular}

HMW, high molecular weight; SHBG, sex hormone binding globulin; sOB-R, soluble form of the leptin receptor.

TABLE 3 Spearman partial correlation coefficients between adipose hormones and testosterone and SHBG

\begin{tabular}{|c|c|c|c|c|c|c|c|c|c|c|c|c|}
\hline $\begin{array}{l}\text { HMW } \\
\text { adiponectin }\end{array}$ & $-0.12^{\star \star \star}$ & $0.29^{* \star *}$ & $-0.08^{* *}$ & $0.22^{\star \star \star}$ & $-0.07^{\star *}$ & $0.17^{* * *}$ & $-0.07^{* *}$ & $0.20^{\star \star \star}$ & $-0.06^{\star}$ & $0.22^{\star \star \star}$ & $-0.06^{\star}$ & $0.17^{\text {***}}$ \\
\hline Leptin & $0.17^{\star \star \star}$ & $-0.27^{\star \star \star}$ & $0.08^{\star \star}$ & $-0.05^{\star}$ & $0.06^{\star}$ & 0.01 & $0.07^{\star \star}$ & -0.02 & $0.08^{\star \star}$ & -0.05 & $0.07^{\star \star}$ & -0.02 \\
\hline sOB-R & $-0.10^{\star * *}$ & $0.24^{* * *}$ & $-0.05^{*}$ & $0.17^{\star * *}$ & -0.04 & $0.14^{* * *}$ & -0.04 & $0.13^{\star * *}$ & -0.04 & $0.16^{* * *}$ & -0.02 & $0.10^{* * *}$ \\
\hline
\end{tabular}

HOMA-IR, homeostasis model assessment insulin resistance index; HMW, high molecular weight; SHBG, sex hormone binding globulin; sOB-R, soluble form of the leptin receptor, T, testosterone.

aPartial 1: Adjusted for age, race-ethnicity, menopausal status, physical activity, smoking status, education level, cycle day of blood draw, and study site.

bartial 2: Adjusted for Partial 1 plus fat mass.

cPartial 3: Adjusted for Partial 2 plus HOMA-IR.

dPartial 4: Adjusted for Partial 1 plus waist circumference.

ePartial 5: Adjusted for Partial 2 plus SHBG in the case of T, and T in the case of SHBG.

fPartial 6: Adjusted for Partial 2-all adipose hormones adjusted for each other.

${ }^{\star \star *} P<0.001 ;{ }^{* \star} P<0.01 ;{ }^{*} P<0.05$. 
TABLE 4 Spearman partial correlation coefficients between adipose hormones and testosterone and SHBG, stratified by BMI

\begin{tabular}{|c|c|c|c|c|c|c|}
\hline & \multicolumn{2}{|c|}{$\mathrm{BMI}<30 \mathrm{~kg} / \mathrm{m}^{2}$} & \multicolumn{2}{|c|}{$\mathrm{BMI} \geq 30 \mathrm{~kg} / \mathrm{m}^{2}$} & \multicolumn{2}{|c|}{$P$ value for interaction } \\
\hline HMW adiponectin & -0.05 & $0.22^{\star \star \star}$ & $-0.12^{\star \star}$ & $0.21^{\star \star \star}$ & 0.82 & 0.08 \\
\hline $\mathrm{sOB}-\mathrm{R}$ & -0.06 & $0.19^{\star \star \star}$ & -0.05 & 0.05 & 0.34 & 0.018 \\
\hline
\end{tabular}

HMW, high molecular weight; SHBG, sex hormone binding globulin; SOB-R, soluble form of the leptin receptor, T, testosterone.

Adjusted for age, race-ethnicity, menopausal status, physical activity, smoking status, education level, cycle day of blood draw, study site, and fat mass.

${ }^{* * *} P<0.001 ;{ }^{* *} P<0.01 ;{ }^{*} P<0.05$.

contribute to leptin resistance and, in part, determine the extent of leptin's effects; sOB-R is thought to delay leptin clearance, increasing circulating leptin concentrations, but decreasing the availability of biologically active leptin, which is the free rather than bound form (24). Despite this, the association between SHBG and sOB-R was independent of leptin levels, suggesting that sOB-R may have associations with biological factors through means other than its regulation of leptin levels. SHBG and sOB-R may be linked through liver-specific pathways; although in fairly low levels, the short form of the leptin receptor has been observed in liver tissue, adjacent to the hepatic vessels (25) and, as we noted above, the liver is the site of SHBG production.

Although statistically significant, we found much weaker associations between testosterone and adipose hormones than was observed between SHBG and adipose hormones, especially after adjustment for fat mass, perhaps suggesting lesser biological relevance of testosterone compared with SHBG in relation to adipose tissue hormone pathways. The vast majority of prior research on the potential regulation of adipose hormones by androgens has occurred in men or in women with PCOS. There have been a limited number of investigations into the associations between androgens and adiponectin in healthy women, and results have been inconsistent, including reports of both negative and positive associations, as well as null findings (20-24,26-29). The reasons for these discrepancies are unclear. Adiponectin is a multimeric complex, forming trimers (low molecular weight), hexamers (medium molecular weight), and multimers (HMW). In human plasma, the low molecular weight and HMW forms are the most abundant, and the HMW form of adiponectin is suggested to be the most biologically active form (30). All but one of the cited studies reported relationships between testosterone and total adiponectin, with each of these reporting null or positive associations. The single study reporting associations with the HMW adiponectin form was the only study to demonstrate a negative association with testosterone similar to our findings, but found it only with the ratio of HMW to total adiponectin, rather than with raw HMW adiponectin levels, alone, as we report (26). Therefore, the failure of most prior studies to examine the HMW adiponectin form, specifically, may partially underlie discrepant results.

In contrast to contradictory findings of an association between testosterone and HMW adiponectin, the positive relationship we report between testosterone and leptin is consistent with prior findings among healthy women $(10,27,31,32)$. To our knowledge, only one other published report has examined the relationship between circulating testosterone and sOB-R levels, finding the same negative relationship we saw, even after adjustment for fat mass among a sample of adolescents and young adults (33).

Although associations between testosterone and adipose tissue hormones were fairly weak, the presence of androgen receptors in both adipocytes and preadipocytes supports the possibility of a regulatory relationship between testosterone and adipose tissue hormones (17). Androgen receptor density is greater in visceral compared with subcutaneous fat (17). Given that visceral adipose tissue secretes pro-inflammatory adipokines in greater quantity, and anti-inflammatory adiponectin in lesser quantity than subcutaneous adipose tissue $(34,35)$, it may be that testosterone binding to visceral adipocytes initiates adverse changes to the hormone secretion profile of adipose tissue. In accordance with the negative association we report between testosterone and HMW adiponectin, testosterone administration has been shown to decrease adiponectin secretion, due to the enhanced intracellular retention of the HMW adiponectin multimer, specifically (36). However, in contrast to the positive association we document between testosterone and leptin, testosterone exposure suppressed leptin gene expression in human adipocytes (37).

It is also possible that the cross-sectional associations we document here represent regulation of testosterone and SHBG levels by adipose hormones. Conversion of androgens to estrogens via aromatization occurs in adipose tissue. However, again possible mechanisms support a negative, rather than positive, feedback loop between leptin and testosterone; leptin may stimulate aromatase expression and depending on the precursor, decrease circulating androgen levels (38). As regards regulation of SHBG by adipose hormones, in addition to regulation by testosterone and estradiol, both leptin and adiponectin receptors have been documented in the liver, the site of SHBG production $(25,39)$.

Prior published data suggest effect modification of sex hormoneadipose hormone relationships by body size. A synergistic relationship was described for free testosterone and waist:hip ratio associations with HMW adiponectin among women with PCOS, whereby women with both high free testosterone and high waist:hip ratio had the lowest HMW adiponectin values (9). For leptin, positive associations between total and free testosterone have been reported in nonobese, whereas null or negative associations are reported in obese $(8,10)$. We found evidence of effect modification of the associations between SHBG and both leptin and sOB-R by body size; SHBG was negatively associated with leptin and positively associated with sOB-R in nonobese, but positively associated with leptin, and not associated with sOB-R in obese. The opposing 
direction of the association between SHBG and leptin in obese vs. nonobese individuals may relate to the loss of leptin sensitivity in obese individuals; High leptin levels in obese individuals are thought to be due to leptin resistance, while in nonobese individuals they may represent functional leptin involved in appropriate feedback responses. Regarding sOB-R, the null association in obese may also relate to leptin resistance, whereby dysregulation of the leptin system results in loss of association between leptin receptor levels and regulators of leptin levels. Alternatively, should the current crosssectional associations represent regulatory relationships, the null relationship between SHBG and SOB-R in obese women may also represent a threshold effect of either SHBG or sOB-R; both SHBG and sOB-R levels were substantially lower in obese vs. nonobese women in the current study and may have been too low to elicit any change in levels of the other.

The current analyses also identified effect modification of SHBG and $\mathrm{T}$ associations with leptin and $\mathrm{sOB}-\mathrm{R}$, respectively, by raceethnicity, finding the strongest associations among Japanese women. The biological explanation for this finding is unclear. As these are cross-sectional analyses and temporality cannot be determined, this finding may represent either a stronger influence of testosterone and SHBG on leptin and sOB-R in Japanese women, or a stronger influence of leptin and sOB-R on testerone and SHBG in Japanese women. In consideration of the first temporal sequence specified above, abdominal visceral adipose tissue has been shown to have a greater density of androgen receptors than subcutaneous adipose tissue (17). However, Japanese women have strikingly lower waist circumference values than the other race-ethnic groups represented in SWAN, and therefore, the lesser abdominal adiposity in Japanese women might be expected to attenuate the influence of androgens on adipokines, rather than strengthen it. In reference to the opposite temporal sequence, namely leptin and sOB-R possibly influencing testosterone and SHBG more strongly in Japanese women, the Japanese women in SWAN have the lowest BMI, even lower than the Chinese SWAN women. Given that leptin sensitivity is proportional to body size, it is conceivable that Japanese women are more sensitive to leptin than the remaining race-ethnic groups. As a result, the influence of leptin and sOB-R on testosterone and SHBG may be greater in Japanese women, as reflected in our finding of stronger associations among Japanese women. Although interaction analyses were adjusted for fat mass, which should conceivably statistically account for this effect, it may be that residual confounding by adiposity remained. Given the number of significance tests performed, these interaction results should be evaluated with extreme caution and require confirmation by future studies.

The results of the current report must be viewed within the context of the limitations of the study. We did not measure free testosterone. This is a cross-sectional study, and therefore, directionality cannot be inferred. However, this study had a number of strengths. Data from three adipose hormones were available across a wide range of BMI. In addition, women of varying menopausal status and ethnicity were included, and a number of possible confounders not previously accounted for were included, most importantly HOMA-IR and waist circumference. Finally, we accounted for adiposity via fat mass, rather than BMI. BMI cannot distinguish between fat mass and muscle mass, and therefore, can be a poor proxy for adiposity particularly in younger and older populations where the degree of muscle mass may vary substantially between individuals or is changing within individuals over time. In the current study, results were similar whether BMI or fat mass was used, suggesting that in these midlife women, muscle mass was likely not significantly confounding the ability of BMI to approximate fat mass.

In conclusion, these cross-sectional results suggest that unfavorable adipose hormone levels are seen with higher testosterone and lower SHBG levels in these midlife women, independent of fat mass and waist circumference. It is known that individuals of similar body size can have very different cardiometabolic profiles, potentially explained in part by different adipose tissue distribution patterns, receptor densities, or secretory profiles in adipose tissue of different individuals (40). These data support the possibility that SHBG, and less likely, though also possible, testosterone, may play a role in determining the particular hormonal expression profile of adipose tissue of a given midlife woman. Therefore, alterations in adipose hormones resulting from alterations in the androgen/estrogen balance determined by SHBG, independent of increases in body fat, may partially explain enhanced susceptibility to metabolic and cardiovascular outcomes with the menopause transition, and similarly, that reduced SHBG levels, independently of any influence on androgen or estrogen levels, further exacerbate disease susceptibility in women during midlife. These intriguing data underscore the need for assessment of the longitudinal relationships between androgens, SHBG, and adipokines.

\section{Supplementary Material}

Supplementary material is linked to the online version of the paper at http://www.nature.com/oby $\mathrm{O}$

\section{Acknowledgments}

The Study of Women's Health Across the Nation (SWAN) has grant support from the National Institutes of Health (NIH), DHHS, through the National Institute on Aging (NIA), the National Institute of Nursing Research (NINR) and the NIH Office of Research on Women's Health (ORWH) (Grants NR004061; AG012505, AG012535, AG012531, AG012539, AG012546, AG012553, AG012554, AG012495). Analysis of the adipose hormone data are from the SWAN Repository (AG017719) and from SWAN ancillary grant HL086858 to Dr. Wildman. The content of this manuscript is solely the responsibility of the authors and does not necessarily represent the official views of the NIA, NINR, NHLBI, ORWH, or the NIH. Clinical Centers: University of Michigan, Ann Arbor - MaryFran Sowers, PI; Massachusetts General Hospital, Boston, MA - Joel Finkelstein, PI 1999 - present; Robert Neer, PI 1994 - 1999; Rush University, Rush University Medical Center, Chicago, IL - Howard Kravitz, PI 2009 - present; Lynda Powell, PI 1994 - 2009; University of California, Davis/Kaiser - Ellen Gold, PI; University of California, Los Angeles - Gail Greendale, PI; Albert Einstein College of Medicine, Bronx, NY - Rachel Wildman, PI 2010 - present; Nanette Santoro, PI 2004 - 2010; University of Medicine and Dentistry - New Jersey Medical School, Newark - Gerson Weiss, PI 1994 - 2004; and the University of Pittsburgh, Pittsburgh, PA - Karen Matthews, PI. NIH Program Office: National Institute on Aging, Bethesda, MD - Sherry Sherman 1994 - present; Marcia Ory 1994 - 2001; National Institute of Nursing Research, Bethesda, MD Program Officers.Central Laboratory: University of Michigan, Ann Arbor - Daniel McConnell (Central Ligand Assay Satellite Services). SWAN Repository: University of Michigan, Ann Arbor - MaryFran 
Sowers.Coordinating Center: University of Pittsburgh, Pittsburgh, PA Kim Sutton-Tyrrell, PI 2001 - present; New England Research Institutes, Watertown, MA - Sonja McKinlay, PI 1995 - 2001. Steering Committee: Susan Johnson, Current Chair. Chris Gallagher, Former Chair. We thank the study staff at each site and all the women who participated in SWAN.

\section{(C) 2012 The Obesity Society}

\section{References}

1. Moschos S, Chan JL, Mantzoros CS. Leptin and reproduction: a review. Fertil Steril 2002;77:433-444.

2. Cnop M, Havel PJ, Utzschneider KM et al. Relationship of adiponectin to body fat distribution, insulin sensitivity and plasma lipoproteins: evidence for independent roles of age and sex. Diabetologia 2003;46:459-469.

3. Rosenbaum M, Nicolson M, Hirsch $\mathbf{J}$ et al. Effects of gender, body composition, and menopause on plasma concentrations of leptin. J Clin Endocrinol Metab 1996; 81:3424-3427.

4. Page ST, Herbst KL, Amory JK et al. Testosterone administration suppresses adiponectin levels in men. $J$ Androl 2005;26:85-92.

5. Sih R, Morley JE, Kaiser FE et al. Testosterone replacement in older hypogonadal men: a 12-month randomized controlled trial. J Clin Endocrinol Metab 1997;82: 1661-1667.

6. Remsberg KE, Talbott EO, Zborowski JV, Evans RW, McHugh-Pemu K. Evidence for competing effects of body mass, hyperinsulinemia, insulin resistance, and androgens on leptin levels among lean, overweight, and obese women with polycystic ovary syndrome. Fertil Steril 2002;78:479-486.

7. Toulis KA, Goulis DG, Farmakiotis D et al. Adiponectin levels in women with polycystic ovary syndrome: a systematic review and a meta-analysis. Hum Reprod Update 2009;15:297-307.

8. Söderberg S, Olsson T, Eliasson $\mathrm{M}$ et al. A strong association between biologically active testosterone and leptin in non-obese men and women is lost with increasing (central) adiposity. Int J Obes Relat Metab Disord 2001;25:98-105.

9. O'Connor A, Phelan N, Tun TK et al. High-molecular-weight adiponectin is selectively reduced in women with polycystic ovary syndrome independent of body mass index and severity of insulin resistance. J Clin Endocrinol Metab 2010;95: 1378-1385.

10. Alexander C, Cochran CJ, Gallicchio L et al. Serum leptin levels, hormone levels, and hot flashes in midlife women. Fertil Steril 2010;94:1037-1043.

11. Sowers MF, Crawford S, Sternfeld B et al. Design, survey sampling and recruitment methods of SWAN: a multi-ceter, multi-ethnic community-based cohort study of women and the menopausal transition. In: Lobos R, Marcus R, Kelsey JL (eds) Menopause: Biology and Pathobiology. Academic Press: San Diego; 2000, pp 17588.

12. Lukaski HC, Bolonchuk WW. Estimation of body fluid volumes using tetrapolar bioelectrical impedance measurements. Aviat Space Environ Med 1988;59: 1163-1169.

13. Boulier A, Fricker J, Thomasset AL, Apfelbaum M. Fat-free mass estimation by the two-electrode impedance method. Am J Clin Nutr 1990;52:581-585.

14. Chumlea WC, Guo SS, Kuczmarski RJ et al. Body composition estimates from NHANES III bioelectrical impedance data. Int J Obes Relat Metab Disord 2002;26: 1596-1609.

15. Sternfeld B, Ainsworth BE, Quesenberry CP. Physical activity patterns in a diverse population of women. Prev Med 1999;28:313-323.

16. Little RJA, Rubin DB. Statistical Analysis with Missing Data, 2nd edn, John Wiley: New York, 2002

17. Dieudonne MN, Pecquery R, Boumediene A, Leneveu MC, Giudicelli Y. Androgen receptors in human preadipocytes and adipocytes: regional specificities and regulation by sex steroids. Am J Physiol 1998;274:C1645-C1652.

18. Anderson DC. Sex-hormone-binding globulin. Clin Endocrinol (Oxf) 1974;3:69-96.
19. Rosner W, Hryb DJ, Kahn SM, Nakhla AM, Romas NA. Interactions of sex hormone-binding globulin with target cells. Mol Cell Endocrinol 2010;316:79-85.

20. Tworoger SS, Mantzoros C, Hankinson SE. Relationship of plasma adiponectin with sex hormone and insulin-like growth factor levels. Obesity (Silver Spring) 2007; $15: 2217-2224$

21. Sieminska L, Wojciechowska C, Niedziolka D et al. Effect of postmenopause and hormone replacement therapy on serum adiponectin levels. Metab Clin Exp 2005; $54: 1610-1614$

22. Yasui T, Tomita J, Miyatani Y et al. Associations of adiponectin with sex hormone-binding globulin levels in aging male and female populations. Clin Chim Acta 2007;386:69-75.

23. Onat A, Hergenç G, Dursunoglu D et al. Relatively high levels of serum adiponectin in obese women, a potential indicator of anti-inflammatory dysfunction: relation to sex hormone-binding globulin. Int J Biol Sci 2008;4:208-214.

24. Zastrow O, Seidel B, Kiess W et al. The soluble leptin receptor is crucial for leptin action: evidence from clinical and experimental data. Int $J$ Obes Relat Metab Disord 2003;27:1472-1478.

25. Hoggard N, Mercer JG, Rayner DV et al. Localization of leptin receptor mRNA splice variants in murine peripheral tissues by RT-PCR and in situ hybridization. Biochem Biophys Res Commun 1997;232:383-387.

26. Merki-Feld GS, Imthurn B, Rosselli M, Spanaus K. Serum concentrations of high-molecular weight adiponectin and their association with sex steroids in premenopausal women. Metab Clin Exp 2011;60:180-185.

27. Laughlin GA, Barrett-Connor E, May S. Sex-specific association of the androgen to oestrogen ratio with adipocytokine levels in older adults: the Rancho Bernardo Study. Clin Endocrinol (Oxf) 2006;65:506-513.

28. Laughlin GA, Barrett-Connor E, May S. Sex-specific determinants of serum adiponectin in older adults: the role of endogenous sex hormones. Int J Obes (Lond) 2007;31:457-465.

29. Rolland YM, Perry HM $3^{\text {rd }}$, Patrick P, Banks WA, Morley JE. Leptin and adiponectin levels in middle-aged postmenopausal women: associations with lifestyle habits, hormones, and inflammatory markers-a cross-sectional study. Metab Clin Exp 2006; 55:1630-1636.

30. Kobayashi H, Ouchi N, Kihara S et al. Selective suppression of endothelial cell apoptosis by the high molecular weight form of adiponectin. Circ Res 2004;94: e27-e31.

31. Lambrinoudaki I, Christodoulakos G, Panoulis C et al. Determinants of serum leptin levels in healthy postmenopausal women. J Endocrinol Invest 2003;26:1225-1230.

32. Isidori AM, Strollo F, Morè $\mathrm{M}$ et al. Leptin and aging: correlation with endocrine changes in male and female healthy adult populations of different body weights. J Clin Endocrinol Metab 2000;85:1954-1962.

33. Chan JL, Blüher S, Yiannakouris $\mathrm{N}$ et al. Regulation of circulating soluble leptin receptor levels by gender, adiposity, sex steroids, and leptin: observational and interventional studies in humans. Diabetes 2002;51:2105-2112.

34. Fain JN, Madan AK, Hiler ML, Cheema P, Bahouth SW. Comparison of the release of adipokines by adipose tissue, adipose tissue matrix, and adipocytes from visceral and subcutaneous abdominal adipose tissues of obese humans. Endocrinology 2004; $145: 2273-2282$.

35. Fisher FM, McTernan PG, Valsamakis G et al. Differences in adiponectin protein expression: effect of fat depots and type 2 diabetic status. Horm Metab Res 2002; 34:650-654

36. Xu A, Chan KW, Hoo RL et al. Testosterone selectively reduces the high molecular weight form of adiponectin by inhibiting its secretion from adipocytes. $J$ Biol Chem 2005;280:18073-18080.

37. Wabitsch M, Blum WF, Muche R et al. Contribution of androgens to the gender difference in leptin production in obese children and adolescents. $J$ Clin Invest 1997; 100:808-813.

38. Catalano S, Marsico S, Giordano C et al. Leptin enhances, via AP-1, expression of aromatase in the MCF-7 cell line. J Biol Chem 2003;278:28668-28676.

39. Civitarese AE, Jenkinson CP, Richardson D et al. Adiponectin receptors gene expression and insulin sensitivity in non-diabetic Mexican Americans with or without a family history of Type 2 diabetes. Diabetologia 2004;47:816-820.

40. Wildman RP. Healthy obesity. Curr Opin Clin Nutr Metab Care 2009;12:438-443. 